\section{Esophageal Intramural Pseudodiverticulosis: A Characteristically Unusual Path to Diagnosis}

We report here a case of a 56-year-old man with chronic alcoholism, who had been suffering from dysphagia and weight loss of more than $20 \mathrm{~kg}$ in the previous six months. Endoscopy showed a stricture at $22 \mathrm{~cm}$ from the dental line, with a lumen of $4 \mathrm{~mm}$. which could not be passed through endoscopically (Olympus GIF-PI0). There was little inflammation of the visible mucosa, suggesting that a malignant tumor might be found underneath. Multiple biopsies taken during two consecutive endoscopies only showed Candida infection, without any sign of malignancy. On a barium swallow, a 9-cm long stricture was documented in the upper portion of the esophagus, without any suspicious alterations. Nor did the CT scan of the thorax reveal any findings suggesting a tumor. To achieve closer inspection of the stenotic area, dilation was carried out using the Savary-Gilliard method, with bougies of up to $12.8 \mathrm{~mm}$ in diameter. A normal-caliber endoscope (Olympus GIF-Q30) was then passed through the stricture, which showed the typical signs of intramural pseudodiverticulosis within the narrow segment (Figure 1). The following barium swallow filled typical sacculations at the site of the stricture (Figure 2). Manometrically, in response to a water bolus, a flattened gradient of esophageal contractions was documented. After a series of dilations and a course of oral antimycotic treatment, the patient's dysphagia ceased, and he put on weight after eight weeks.

Esophageal intramural pseudodiverticulosis is a rare condition characterized by tiny, flask-shaped sacculations of the esophageal wall. Since its first description by Mendl et al. (1), about 120 cases have been reported in the international literature. The matrix of the pseudodiverticula has been shown to be pathologically dilated excretory ducts of the esophageal submucous glands (2). There has been much speculation about the etiology and pathogenesis of this condition $(3-5)$. The ease reported here illustrates a characteristically unusual diagnostic approach, eventually leading to the diagnosis of this rare disease.

\section{A. Gillessen, J. Konturek, N. Roos, W. Domschke}

Dept. of Medicine B, University of Münster. Münster, Germany

\section{References}

I. MendI K. McKay JM, Tanner $\mathrm{CH}$. Intramural diverticulosis of the oesophagus and Rokitansky-Aschoff sinuses in the gallbladder. Br J Radiol 1960; 33: 496-501.

2. Umlas J, Sakhuja R. The pathology of esophageal intramural pseudodiverticulosis. Am J Clin Pathol 1976; 65:314-20.

3. Sabanathan S, Salama FD, Morgan WE, Oesophageal intramural pseudodiverticulosis. Thorax 1985; 40: 849-57.

4. Heinze W, Beges W, Borchard F, et al. Oesophageal intramural pseudodiverticulosis: clinical aspects, morphological changes and functional disorders. Dtsch Med Wochenschr 1985; 110: $1077 \quad 83$

5. Levine MS, Moolten DN, Herlinger H, et al. Esophageal intramural pseudodiverticulosis: a reevaluation. Am J Radiol 1986: 147: 1165-70.

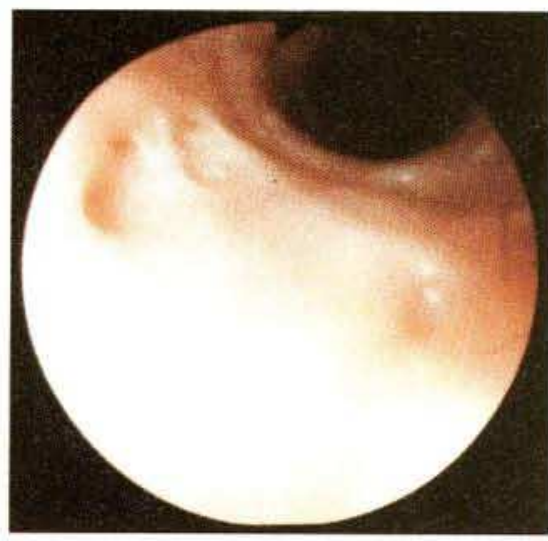

Figure 1: Endoscopy of the esophagus, demonstrating numerous openings in the esophageal wall

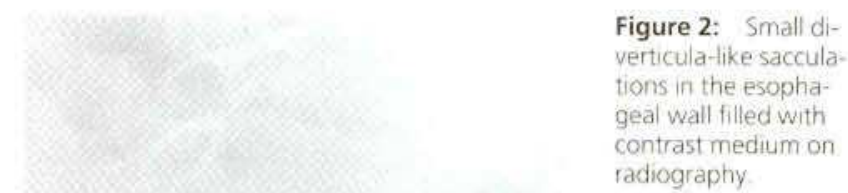

Corresponding Author

A. Gillessen, M. D.

Dept. of Medicine B

Universität Münster

Albert-Schweitzer-Strasse 33

48149 Münster

Germany

Fax: $+49-251-837576$ 